\title{
Low-cost orientation system for a concentrating solar collector with static reflector and tracking receiver
}

\author{
V. Martinez ${ }^{1}$, B. Alorda ${ }^{2}$, A. Moia ${ }^{1}$ and R. Pujol ${ }^{1}$ \\ Departament of Physics \\ ${ }^{1}$ Mechanical Engineering Group \\ ${ }^{2}$ Electronic Technology Group \\ Universitat Illes Balears \\ Ctra. Valldemossa, km 7.5 Palma de Mallorca \\ phone: +34 971 171374, fax: +34 97117 3426, e-mail: victor.martinez@uib.es
}

\section{Brief introduction}

A solar linear concentrator with a fixed reflector and a moving focus is under development. The main objective of the research is to develop a low - cost, roof integrated, solar collector suited for mid - range thermal applications, such as absorption cooling and industrial heat $\left(90-200{ }^{\circ} \mathrm{C}\right)$. This range of temperatures could be achieved with low concentration factors (9 - 15 suns).

This range of radiation concentration is also well suited for the development of Combined Solar Heat and Power (CHP) systems integrating PV and thermal converters in the same unit [1]. Recently, this kind of application has gained much attention from the solar energy industry with several interesting development projects from both the industry and the academy [2].

The proposed solution is based on a static reflector with a moving linear focus (Figure 1). The basic geometric concepts were developed in the 70 s [3] but the product development never passed beyond the experimental prototype stage.

Nevertheless, a detailed ray-tracing modelling of the reflector behaviour shows clearly that it is possible to achieve a high optical efficiency into the target concentration range.

Although the reflector is static, the sun path should be tracked by the receiver to be kept on the reflector focus. Therefore a precise and inexpensive tracking control system is of fundamental importance for a successful development of the collector.

Keywords: Solar tracking, Low-cost system, Modular control system.

\section{The solar collector}

The solar collector is composed of a fixed reflector with a linear focus that follows a circular path. Thus, in order to keep the moving receiver on the right position is enough to mount it on the tip of an articulated arm which angle is adjusted according to the sun position (Figure 2.)
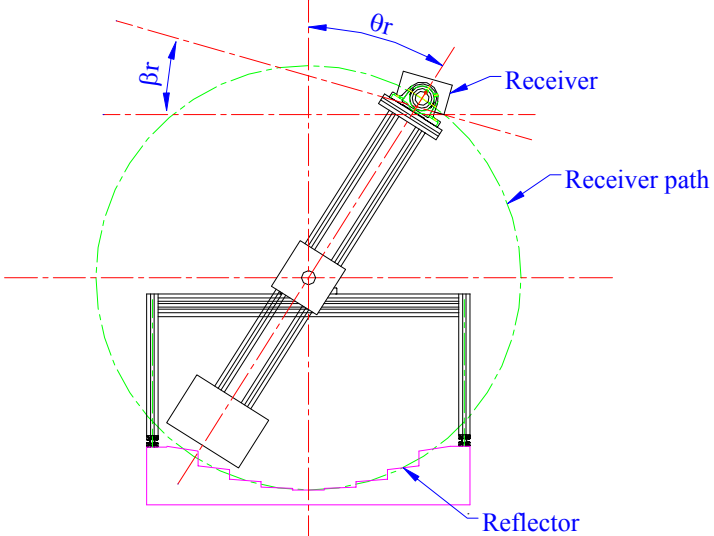

Figure 1. Tracking structure

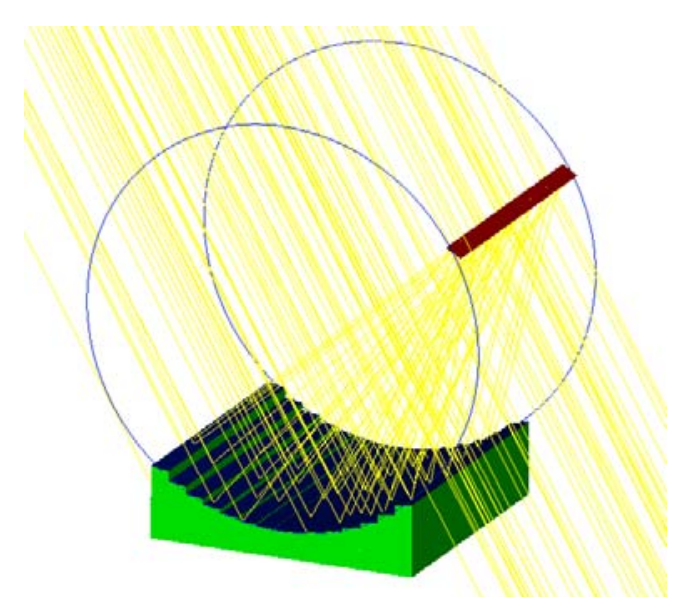

Figure 2. Solar rays projection simulation.

The exact angle at which the receiver should be placed depends mainly on two factors:

1. The sun's position

2. The theoretical orientation of the reflector 
The first parameter can be accurately predicted given the coordinates of the location where the collector is installed and an accurate time reference. The problems arise when the angles relative to the collector are to be estimated, because, although it is possible to predict theoretically the relative sun angle given the orientation of the collector and the sun's position, in practice this prediction is likely to be inaccurate mainly due to the errors in the on-site determination of the orientation of the collector. Furthermore it is likely that small differences could be found between collectors placed into the same array and should, in theory, share the same orientation.

Another factor that would influence the design of the control system of the tracking mechanism, is that, depending on the mirror design, the radiation distribution on the receiver could be highly non uniform. Therefore, in those cases, it is likely that the maximum energy output is not going to be found at the theoretical position, but somewhat displaced in the direction of the highest energy peak. (see Figure 3)

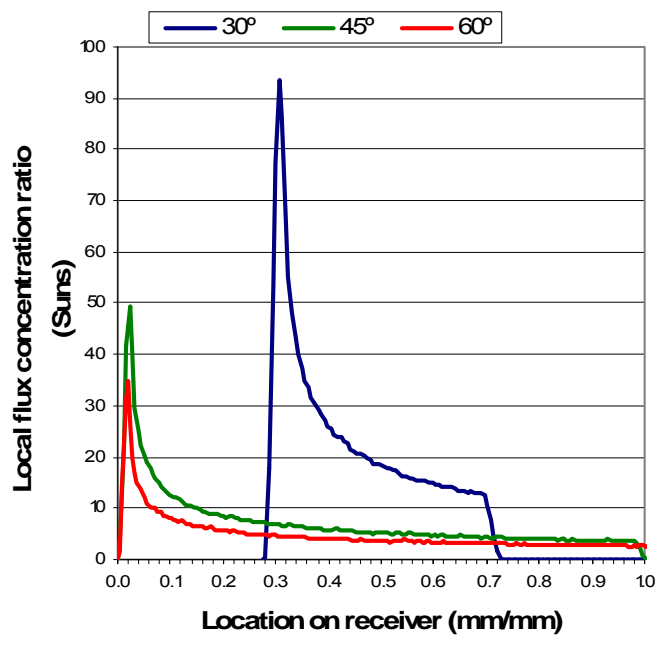

Figure 3. Radiation distribution for a parabolic reflector

\section{Angular requirements}

The receiver tracks the sun moving on a circle path (see Figure 1). It's needed to know the sun vector to place the receiver in the right position. The sun vector is determinate by the two solar angles, $\theta_{S}$ and $\gamma_{S}$, (see Fig. 5) referred to any local position of the earth. We used the algorithm described by Duffie and Beckman [4] to determine these angles. The parameters that are needed for the algorithm are: local latitude, local longitude, day of the year and local hour. Once the solar angles referred to the North - South and Zenith axis have been determined by the algorithm, it's necessary to transform those angles to the collector local axis defined in the figure 4 , obtaining $\theta_{c}$ and $\gamma_{c}$.

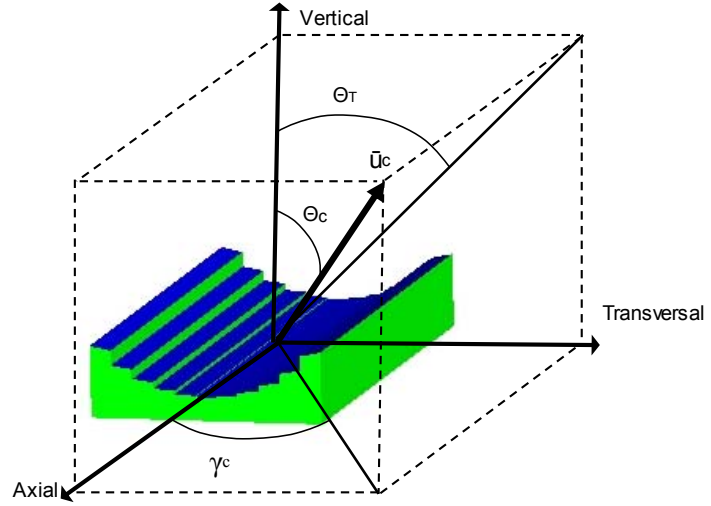

Figure 4. Solar angles referred to the collector.

A transformation, $T$, to calculate the sun vector referred to the collector, $\vec{u}_{c}$, is used:

$$
\vec{u}_{c}=T \vec{u}_{s}
$$

Where $\vec{u}_{S}$ is the sun vector and the transformation $T$ depends of the orientations angles of the collector. These angles are $\gamma, \alpha, \beta$. Where $\gamma$ is the collector azimuth angle, $\alpha$ is the transversal collector slope angle and $\beta$ is the axial collector slope angle. Figure 5 shows a sloped collector and the orientation angles.

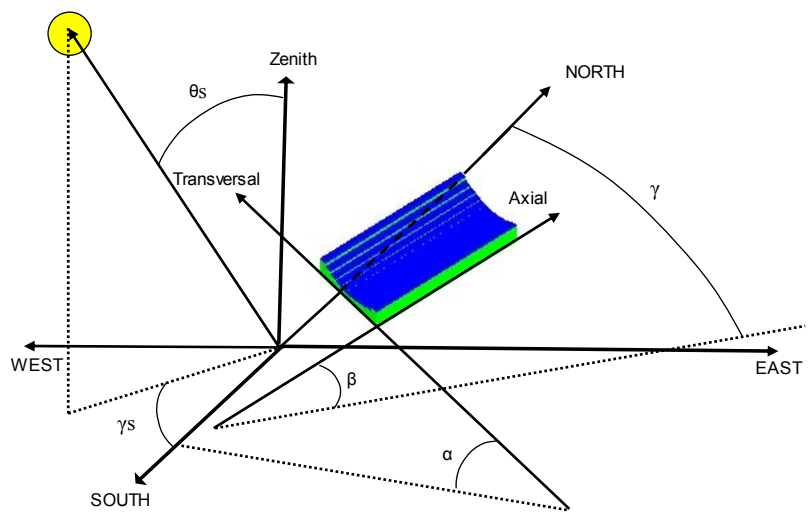

Figure 5. Orientation angles of the collector and solar angles.

The position of the receiver depends of the incident transversal angle named $\theta_{T}$, see Figure 4. As $\theta_{T}$ is the angle between the projected sun vector to the transversal plane and the $Z$ axis it's easy to show that the relationship of $\theta_{T}$ with zenith and azimuth angles $\left(\theta_{c}\right.$ and $\left.\gamma_{c}\right)$ referred to the collector is:

$$
\theta_{T}=\arctan \frac{\sin \theta_{c} \sin \gamma_{c}}{\cos \theta_{c}}
$$

To put the receiver in the right position it's necessary to place the arm of the receiver in an angle $\theta_{r}$ and to tilt the receiver an angle $\beta_{r}$. Both angles depend of the transversal incidence angle $\theta_{T}$. The relationship of those angles with $\theta_{T}$ is given by:

$$
\begin{gathered}
\theta_{r}=2 \theta_{T} \\
\beta_{r}=\theta_{T}
\end{gathered}
$$


The flow diagram of the algorithm required to track the sun is shown in Figure 6.

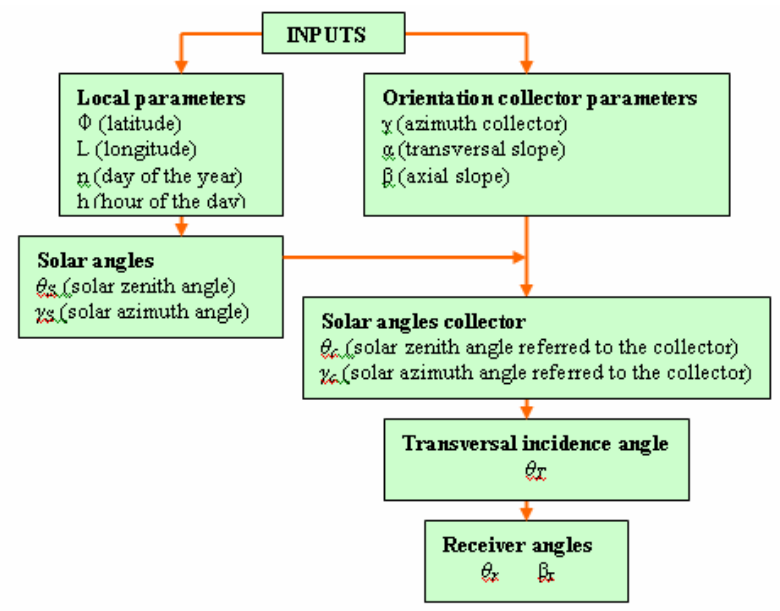

Figure 6. Inputs and relationship of the different parameters.

\section{System Architecture}

The proposed system approach is designed to control the orientation of a high number of collectors. The controller system is divided in two levels: first-level is the main-frame and contains the configuration data. Second-level is the orientation controller that sets the position of each positioning mechanism to the correct angle.

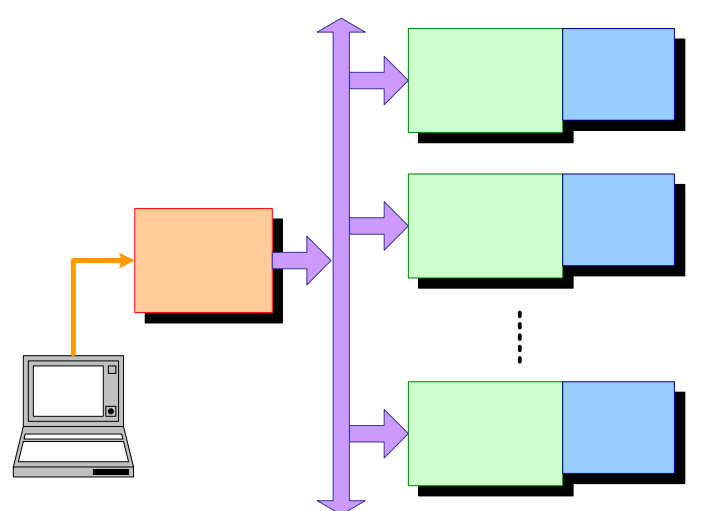

Figure 7. System architecture scheme.

Figure 7 shows the complete architecture. The mainframe level is connected to each orientation controller using the CAN protocol to obtain a good reliability, while the main-frame is connected to a computer using a RS232 bus.

The connection to computer is needed to initialize the main-frame and allows obtaining information about the present estate of system. The same connection can be used for statistical data collection and a USB connection can be used for high volume of data.

\subsection{The Main board approach}

The main-frame board is based on a PIC microcontroller using a real-time circuit to obtain accurate time evolution.
The functions implemented at this level are:

- Mathematical angle estimation.

- Maintain the real time and date.

- Communicate the angle estimation to each orientation controller boards.

- Maintain actualized information for plug-andplay function using CAN bus.

- Permit communications with an external computer

- Save the configuration data: earth position, solar orientation ...

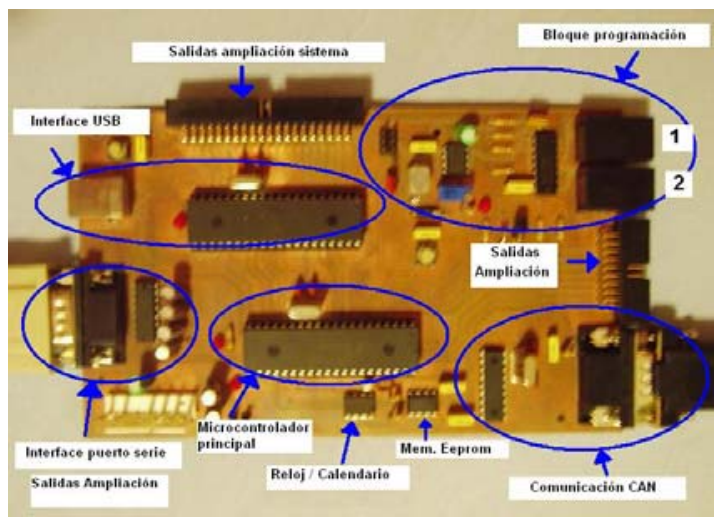

Figure 8. Main-frame board

Figure 8 shows the main-frame board implemented to accomplish these functions. The board develops all functions and has prevision for future applications.

The board has two microcontrollers: the main controller is used for mathematical angle estimation and configuration maintenance. The angle estimation is calculated using a RTC device based on a quartz crystal. This device permits successful theoretical angle estimation due to good real time resolution. Another advantage of using RTC device is when power is off. The real time is maintained thought an auxiliary-battery. Since the whole system does not works when power is off, the time and configuration data is saved and maintained. The system configuration is saved in an external EEPROM allowing maintaining the configuration of the system until the power is on again.

The second microcontroller is used for communication purposes and offers high capabilities for future applications commented in the conclusions section.

\subsection{The control board approach}

The orientation controller board is also based on a PIC microcontroller. The orientation motor is controlled using a transistor bridge circuit and the position of the motor is measured using an encoder. The position feedback information permits orientating the focus to the estimate angle received though CAN bus [5] from mainframe with a precision of $0.04^{\circ}$.

The functions implemented with this board are:

- Receive from CAN bus the new estimated angle.

- Assure the correct angle using the encoder feedback circuit 
- Actuate on the focus motor to move the captor to correct angle.

- Maintain statistical data using an I2C external EEPROM.

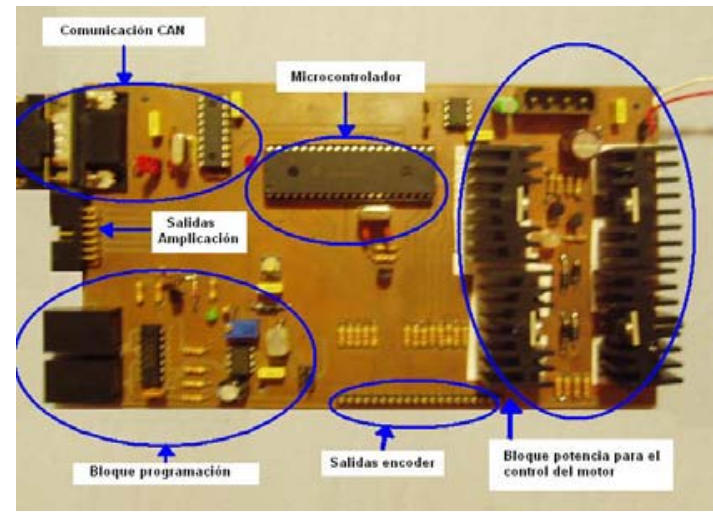

Figure 9. Orientation controller board

Figure 9 shows the orientation controller board where is possible to observe the central position of the PIC microcontroller. The board right side contains the motor driver and the left side contains the programmer and the communication bus.

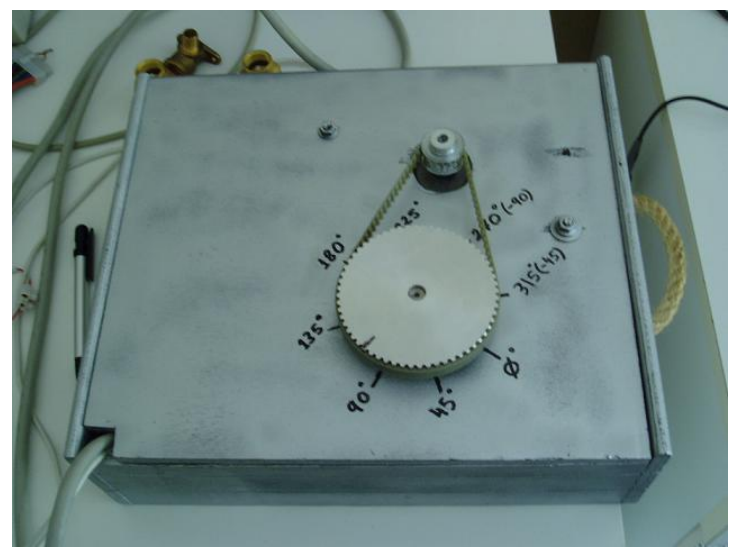

Figure 10. Motor and encoder structure used for experiments

Figure 10 shows an implementation of the motor and encoder components used to verify the correct function of the control system in the laboratory.

The motor rotation is controlled using three different velocities: the faster is used to test the behaviour of system when a new configuration is loaded in the main board and this new data change the estimated angle in a difference of more than $45^{\circ}$.

The middle velocity is used when the system is in the night period (that is, when the rays of sun do not arrive to the receiver) and the receiver is going to initial position to wait for the firsts rays of sunset.

And the lowest velocity is used during day period and adjusts the receiver to the correct angle. This velocity in combination with absolute encoder permits a very good resolution in the final angle.

This board will permit a better adjust of angle using some kind of solar radiation measurements computing the efficiency of collector and introducing local variations in the angle used.

\section{Experimental results}

The system and structure has been installed on the top of an university building using only one collector with one motor. Different angles are been used to calibrate the correct orientation of structure and to introduce some physical characteristics is the motor control program (like time to start to move, resistance to movement, ...). At this point, the system is under efficiency study to determine the best motor control algorithms.

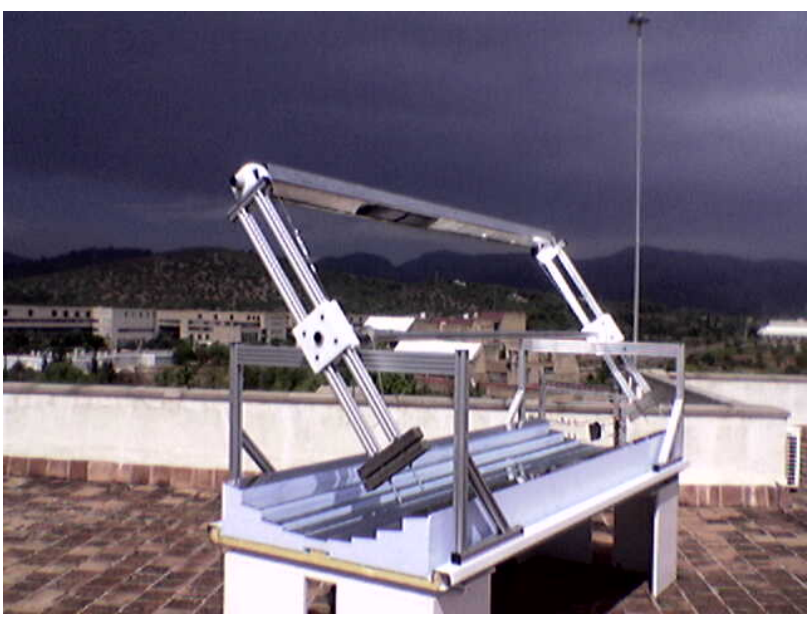

Figure 11. Solar Collector with static reflector

Figure 11 shows the solar collector structure installed outdoor.

\section{Conclusions}

A low-cost orientation system has been developed using commercial components and using standard protocols for communications.

The main advantages obtained with this approach are:

- Very low-cost system

- High grade of scalability, increasing the number of orientation controllers.

- $\quad$ Easy usability for future applications.

- High resolution in the feedback angle measurement.

The specifications accomplished are:

- Correct orientation of mobile focus obtaining a precision of $0.04^{\circ}$

- Development of an independent system with computer communications capabilities.

- Robustness in the communications using an industrial bus.

- Easy programmer system implemented on each board.

The low-cost has been obtained using low-cost components with high quality ratio. 
The system will be improved in future applications introducing:

- Control of real-time and date deviations using international information transmitted by radio.

- High capability of transmitting statistical information using the incorporated USB connection

- Fine adjust of each orientation system using lightsensors or other sensors.

\section{Acknowledgement}

This work was cofunded by The Universitat de les Illes Balears, The Direcció General d'Energia de la Conselleria de Comerç Industria i Energia del Govern de les Illes Balears and by Sistemes vançats d'Energia Solar Tèrmica, SCCL, and we extend our thanks to all of them. Thanks are also due to Víctor García, who implemented this design during his degree thesis.

\section{References}

[1] Rosell J.I., Vallverdú X., Lechón M.A., Ibáñez M, “Design and simulation of a low concentrating photovoltaic/thermal system", Energy Conversion and Management, pp. 3034-3046, 2005.

[2] Knight O; International Solar Energy Society, "Solar CHP, Breaking through the Solar Cost Barrier?", Refocus magazine, pp. 40-42, May/June 2005

[3] Russell J.L., DePlomb E.P., "Concentrator, Method and system for utilizing radiant energy", US patent 3868823

[4] Duffie JA, Beckman WA, Solar Engineering of Thermal Process. New York. John Wiley, 1991 ISBN 0471510564.

[5] Bassi E., Benzi F., Lusetti L. Buja G.S., "Communication protocols for electrical drives", IEEE International Conference on Industrial Electronics, Control and Instrumentation. Vol 1, pp. 706-711, 1995. 